\title{
The Great Observatories Origins Deep Survey
}

\section{VLT/FORS2 spectroscopy in the GOODS-South Field $\star, \star \star$}

\author{
E. Vanzella ${ }^{1,2}$, S. Cristiani ${ }^{2}$, M. Dickinson ${ }^{3}$, H. Kuntschner ${ }^{4}$, L. A. Moustakas ${ }^{5}$, M. Nonino ${ }^{2}$, P. Rosati ${ }^{6}$, \\ D. Stern ${ }^{8}$, C. Cesarsky $^{6}$, S. Ettori ${ }^{6}$, H. C. Ferguson ${ }^{5}$, R. A. E. Fosbury ${ }^{4}$, M. Giavalisco ${ }^{5}$, J. Haase ${ }^{4}$, A. Renzini ${ }^{6}$, \\ A. Rettura ${ }^{6,7}$, P. Serra ${ }^{4,9}$, and the GOODS Team \\ 1 Dipartimento di Astronomia dell'Università di Padova, Vicolo dell'Osservatorio 2, 35122 Padova, Italy \\ e-mail: evanzell@eso.org \\ 2 INAF - Osservatorio Astronomico di Trieste, via G.B. Tiepolo 11, 40131 Trieste, Italy \\ 3 National Optical Astronomy Obs., PO Box 26732, Tucson, AZ 85726, USA \\ 4 ST-ECF, Karl-Schwarzschild Str. 2, 85748 Garching, Germany \\ 5 Space Telescope Science Institute, 3700 San Martin Drive, Baltimore, MD 21218, USA \\ ${ }^{6}$ European Southern Observatory, Karl-Schwarzschild-Strasse 2, Garching 85748, Germany \\ 7 Université Paris-Sud XI, 15 rue Georges Clemenceau, 91405 Orsay Cedex, France \\ 8 Jet Propulsion Laboratory, California Institute of Technology, MS 169-506, 4800 Oak Grove Drive, Pasadena, \\ CA 91109, USA \\ 9 Kapteyn Instituut, Landleven 12, 9747 AD Groningen, The Netherlands
}

Received 25 June 2004 / Accepted 14 December 2004

\begin{abstract}
We present the first results of the ESO/GOODS program of spectroscopy of faint galaxies in the Chandra Deep Field South (CDF-S). 399 spectra of 303 unique targets have been obtained in service mode with the FORS2 spectrograph at the ESO/VLT, providing 234 redshift determinations (the median of the redshift distribution is at 1.04). The typical redshift uncertainty is estimated to be $\sigma_{z} \simeq 0.001$. Galaxies have been color selected in a way that the resulting redshift distribution typically spans from $z=0.5$ to 2 . The reduced spectra and the derived redshifts are released to the community through the ESO web page http://www.eso.org/science/goods/. Large scale structure is clearly detected at $z \simeq 0.67,0.73,1.10$ and 1.61 . Three Lyman-break galaxies have also been included as targets and are confirmed to have redshifts $z=4.800,4.882$ and 5.828. In a few cases, we observe clear [OII] 3727 rotation curves, even at the relatively low resolution $(\mathfrak{R}=860)$ of the present observations. Assuming that the observed velocity structure is due to dynamically-relaxed rotation, this is an indication of large galactic masses (few times $10^{11} M_{\odot}$ ) at $z \sim 1$.
\end{abstract}

Key words. cosmology: observations - cosmology: large-scale structure of Universe - galaxies: evolution

\section{Introduction}

The Great Observatories Origins Deep Survey (GOODS) is a public, multifacility project that aims to answer some of the most profound questions in cosmology: how did galaxies form and assemble their stellar mass? When was the morphological differentiation of galaxies established and how did the Hubble Sequence form? How did AGN form and evolve, and what role do they play in galaxy evolution? How much do galaxies and AGN contribute to the extragalactic background light?

\footnotetext{
* The catalog (Table 2) is also available in electronic form at the CDS via anonymous ftp to

cdsarc.u-strasbg.fr $(130.79 .128 .5)$ or via

http://cdsweb.u-strasbg.fr/cgi-bin/qcat?]/A+A/434/53

$\star \star$ Based on observations made at the European Southern Observatory, Paranal, Chile (ESO programme 170.A-0788, The Great Observatories Origins Deep Survey: ESO Public Observations of the SIRTF Legacy/HST Treasury/Chandra Deep Field South).
}

Is the expansion of the universe dominated by a cosmological constant? A project of this scope requires large and coordinated efforts from many facilities, pushed to their limits, to collect a database of sufficient quality and size for the task at hand. It also requires that the data be readily available to the worldwide community for independent analysis, verification, and follow-up.

The program targets two carefully selected fields, the Hubble Deep Field North (HDF-N) and the Chandra Deep Field South (CDF-S), with three NASA Great Observatories (HST, Spitzer and Chandra), ESA's XMM-Newton, and a wide variety of ground-based facilities. The area common to all the observing programs is $320 \mathrm{arcmin}^{2}$, equally divided between the North and South fields. For an overview of GOODS, see Dickinson et al. (2003), Renzini et al. (2002) and Giavalisco et al. (2004a).

Spectroscopy is essential to reach the scientific goals of GOODS. Reliable redshifts provide the time coordinate needed 
to delineate the evolution of galaxy masses, morphologies, clustering, and star formation. They calibrate the photometric redshifts that can be derived from the imaging data at $0.36-8 \mu \mathrm{m}$. Spectroscopy will measure physical diagnostics for galaxies in the GOODS field (e.g., emission line strengths and ratios to trace star formation, AGN activity, ionization, and chemical abundance; absorption lines and break amplitudes that are related to the stellar population ages). Precise redshifts are also indispensable to properly plan for future follow-up at higher dispersion, e.g., to study galaxy kinematics or detailed spectral-line properties.

The ESO/GOODS spectroscopic program is designed to observe all galaxies for which VLT optical spectroscopy is likely to yield useful data. The program makes full use of the VLT instrument capabilities (FORS2 and VIMOS), matching targets to instrument and disperser combinations in order to maximize the effectiveness of the observations. The magnitude limits and selection bandpasses depend to some degree on the instrumental setup being used. The aim is to reach mag 24-25 with adequate $\mathrm{S} / \mathrm{N}$, with this limiting magnitude being in the $B$ band for objects observed with the VIMOS LRBlue grism, in the $V$ band for those observed in the VIMOS LR-Red grism, and in the $z$ band for the objects observed with FORS2. This is not only a practical limit, however, but is also well matched to the scientific aims of the GOODS program. The ACS $i_{775}$ imaging samples rest-frame optical ( $B$-band) light out to $z=1$, where $i_{775}=25$ reaches 1.5 to 2 mag fainter than $L_{B}^{*}$. This is also the practical limit for high-quality, quantitative morphological measurements from the ACS images (cf. Abraham et al. 1996; Moustakas et al. 2004). Similarly, $i_{775}=$ 25 is $\sim 1$ mag fainter than the measured $L^{*} \mathrm{UV}$ for $z=3$ Lyman Break Galaxies (LBGs), and 0.5 mag fainter than that at $z=4$ (Steidel et al. 1999). These are the limits to which GOODS/SIRTF IRAC data will robustly measure rest-frame near-IR light, and hence constrain the stellar mass.

In this paper we report on the first spectroscopic follow-up campaign in the Chandra Deep Field South (CDF-S), carried out with the FORS2 instrument at the ESO VLT in the period fall 2002-spring 2003 (the first 9 masks, 348 slits). Further 17 masks have been observed during the period 2003 and early 2004, for which the reduction process has started and will be presented elsewhere (Vanzella et al., in preparation).

The paper is organized as follows: in Sect. 2 we describe the target selection and in Sect. 3 the observations and the reduction. The redshift determination is presented in Sect. 4. In Sect. 5 we discuss the data and in Sect. 6 the conclusions are presented. Throughout this paper the magnitudes are given in the $\mathrm{AB}$ system $\left(\mathrm{AB} \equiv 31.4-2.5 \log \left\langle f_{v} / \mathrm{nJy}\right\rangle\right)$, and the ACS $F 435 W, F 606 W, F 775 W$, and $F 850 L P$ filters are denoted hereafter as $B_{435}, V_{606}, i_{775}$ and $z_{850}$, respectively. We assume a cosmology with $\Omega_{\mathrm{tot}}, \Omega_{\mathrm{M}}, \Omega_{\Lambda}=1.0,0.3,0.7$ and $H_{0}=70 \mathrm{~km} \mathrm{~s}^{-1} \mathrm{Mpc}^{-1}$.

\section{Target selection}

Objects were selected as candidates for FORS2 observations primarily based on the expectation that the detection and measurement of their spectral features would benefit from the high throughput and spectral resolution of FORS2, and its reduced fringing at red wavelengths, relative to other instrumental options such as VIMOS. In particular, we expect that the main spectral emission and absorption features for galaxies at $0.8<$ $z<1.6$ would appear at very red optical wavelengths, out to $\sim 1 \mu \mathrm{m}$. Similarly, very faint Lyman break galaxies at $z \gtrsim 4$, selected as $B_{435}, V_{606}$ and $i_{775}$-dropouts from the GOODS ACS photometry, also benefit greatly from the red throughput and higher spectral resolution of FORS2.

In practice, several categories of object selection criteria were used to ensure a sufficiently high density of target candidates on the sky to efficiently fill out multi-slit masks. Using ACS photometry in the AB magnitude system, these criteria were:

1. Primary catalog: $\left(i_{775}-z_{850}\right)>0.6$ and $z_{850}<24.5$. This should ensure redshifts $z \gtrsim 0.7$ for ordinary early-type galaxies (whose strongest features are expected to be absorption lines), and higher redshifts for intrinsically bluer galaxies likely to have emission lines.

2. Secondary catalog: $0.45<\left(i_{775}-z_{850}\right)<0.6$ and $z_{850}<$ 24.5.

3. Photometric-redshift sample: $1<z_{\text {phot }}<2$ and $z_{850}<24.5$, using an early version of GOODS photometric redshifts like those described by Mobasher et al. 2004.

4. $i_{775}$-dropout and $V_{606}$-dropout Lyman break galaxy candidates, selected from the criteria of Dickinson et al. (2004a) and Giavalisco et al. 2004b, respectively.

5. A few miscellaneous objects, including host galaxies of supernovae detected in the GOODS ACS observing campaign.

Target selection and mask design for the 2002-3 GOODS/FORS2 campaign was carried out while the GOODS ACS observations were still in progress, and before final ACS data reduction or cataloging could be completed. The targets were therefore selected based on interim data reductions and catalogs, initially based on only one epoch of ACS imaging, and later using the three-epoch ACS stacks and preliminary catalogs described in Giavalisco et al. (2004a). Because of this, the actual magnitudes and colors of the observed galaxies from the final, 5-epoch ACS image stack, which we report here in Table 2, may not exactly match the intent of the original selection criteria. When designing the masks, we generally tried to avoid observing targets that had already been observed in other redshift surveys of this field, namely, the K20 survey of Cimatti et al. (2002) and the survey of X-ray sources by Szokoly et al. (2004).

399 spectra of 303 unique targets have been extracted from the first nine masks (96 targets have been observed more than one time). Out of these 303 targets, 96 meet the primary catalog, 33 meet the secondary catalog, 43 meet the photometric redshift selection, 28 and 4 the fourth and fifth criteria, respectively. The remaining 99 sources have been serendipitously identified, due to: a) sources randomly in the slit other than the target or b) sources put in the slit in the situation where no targets were available or c) bright objects put in the slit for the pointing of the mask. 
Table 1. Journal of the MXU observations.

\begin{tabular}{lcc}
\hline \hline Mask ID & UT date & Exp. time (s) \\
\hline 990247 & 30 Dec. 2002-2, 6 Jan. 2003 & $12 \times 1200$ \\
984829 & 9 Dec. 2002-3, 4 Jan. 2003 & $12 \times 1200$ \\
985831 & 5 Jan.-4, 7 Feb. 2003 & $15 \times 1200+663$ \\
973934 & 7, 30, 31 Jan. 2003 & $12 \times 1200$ \\
952426 & 6, 7 Jan. 2003 & $12 \times 1200$ \\
981451 & 31 Jan.-24, 27 Feb.-22 Nov.-17 Dec.-30 Jul.-1 Aug. 2003 & $24 \times 1200$ \\
995131 & 5-6 Oct. 2002 & $8 \times 1800$ \\
994852 & 4 Oct. 2002 & $8 \times 1800$ \\
990652 & 8 Dec. 12 Nov. 2002 & $14 \times 1200+300+900$ \\
\hline
\end{tabular}

Out of 43 galaxies selected via photometric redshift, 30 have a spectroscopic redshift identification, only 3 out of 30 have redshift less than 0.9 .

The spectroscopic database presented here is largely incomplete: non of the above listed categories has been exhaustively observed (for example about one third of the objects in class 1 and 2), nor any GOODS subarea has been fully covered.

\section{Observations and data reduction}

The VLT/FORS2 spectroscopic observations were carried out in service mode during several nights in 2002 and 2003. A summary is presented in Table 1 . In all cases the 300I grism was used as dispersing element without order-separating filter. This grism provides a scale of roughly $3.2 \AA /$ pixel. The nominal resolution of the configuration was $R=\lambda / \Delta \lambda=860$, which corresponds to about $9 \AA$ at $8000 \AA$. The spatial scale of FORS2 was $0.126^{\prime \prime} /$ pixel, the slit width was always $1^{\prime \prime}$. Dithering of the targets along the slits was applied typically with steps of $0, \pm 8$ pixels, in order to effectively improve the sky subtraction an the removal of CCD cosmetic defects. The mean shift applied was \pm 8 pixels.

\subsection{Data reduction}

Data were reduced with a semi-automatic pipeline that we have developed on the basis of the MIDAS package (Warmels 1991), using commands of the LONG and MOS contexts (Fig. 1). The frames have been bias-subtracted and flat-fielded. For each slit the sky background was estimated with a second order polynomial fitting. In some cases, better results have been obtained adopting a first order polynomial. The fit has been computed independently in each column inside two windows, above and below the position of the object (if more objects are present in the slit, a suitable modification of the windows is applied).

The resulting dithered, sky-subtracted, two-dimensional frames for each object are then averaged, with the weighting determined based on exposure time, seeing, and meteorlogical conditions. Spatial median filtering has been applied to each dithered exposures to clean the cosmic rays. The FORS2 instrument shows an exquisite response in the red domain (beyond $8000 \AA$ ), in practice no appreciable residual fringe pattern affects the extracted signal. Rather, the sky residuals dominate the noise in the regions where the intensity and the density of the skylines increase (see Fig. 1). The individual dithered sky-subtracted spectra have been visually inspected to verify that the object is indeed in the expected region of the slit. This step is necessary since the applied small spatial offsets between the science exposures can result in objects falling too close to the slit edge or even outside the slit (in exceptional cases). After this visual screening, the spatial offset between different exposures of the same object was calculated on the basis of the world coordinate system (WCS) information stored in the frame headers. The individual exposures were co-added (including the rejection of bad pixels or cosmic ray hits) after applying these spatial shifts. The frames were shifted in the spatial direction and only by integer numbers of pixels. As the objects were sufficiently well sampled (the pixel scale was significantly smaller than the seeing), no significant blurring of the spectra was observed, while the statistical properties of the individual pixels were preserved.

The position of the target on the detector was estimated by collapsing 700 columns in the dispersion direction and measuring the center of the resulting profile. The 1-D object signal was obtained using the "optimal extraction" method of MIDAS. This procedure calculates a weighted average in each column, based on both the estimated object profile and photon statistics. The extraction has been carried out in a window, adapted case by case according to the distortion, the size of the object, etc.

Wavelength calibration was calculated on (daytime) arc calibration frames, using three arc lamps (a He, and two Ar lamps) providing sharp emission lines over the whole spectral range used (6000-10800 $\AA$ ). The object spectra were then rebinned to a linear wavelength scale. We have verified the accuracy of the wavelength calibration by checking the position of 25 narrow skylines in the science exposures (from 5577 to $10400 \AA$ ). Systematic translations of the wavelength scale have been typically measured to be of the order of $\pm 1 \AA$ and corrected. The final (absolute) mean wavelength accuracy for all spectra is $0.9 \pm 0.1 \AA$ rms.

Relative flux calibration was achieved by observations of standard stars listed by Bohlin et al. (1995). Since the standard stars are typically quite blue and second order light can be substantial, the calibration spectra were obtained both with and without order-sorting filters, providing calibration across the entire optical window. As noted previously, we opted to obtain the science target without an order-sorting filter, implying deleterious effects to the flux calibration, particularly for bluer objects and at longer wavelengths. For the red objects which dominated the FORS2 target selection, we felt that the 


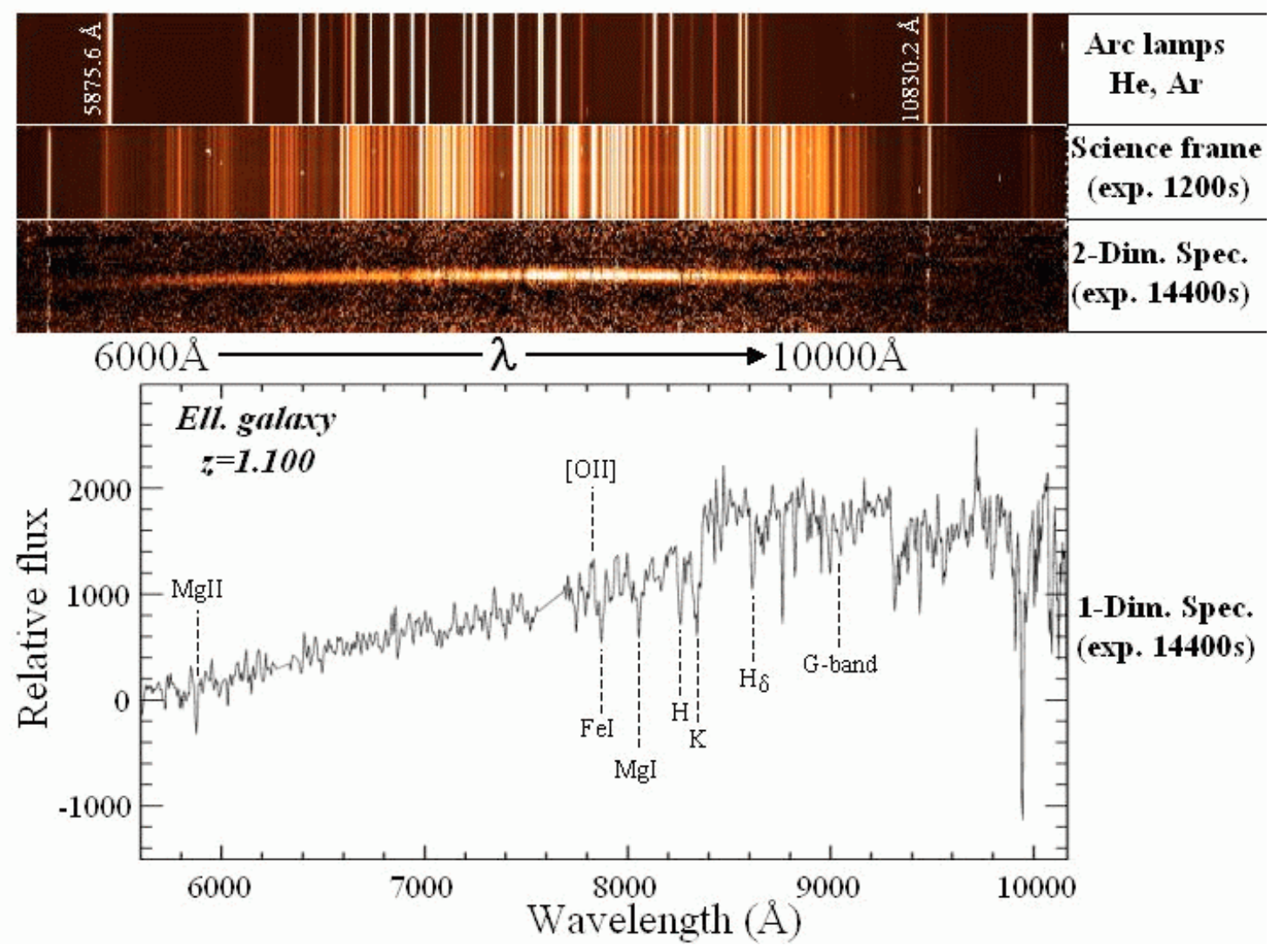

Fig. 1. Typical FORS2 data products for an individual slit of multi-object mask. From the top of the figure: the 2-D spectrum of the arc lines used for the wavelength calibration, a 2-D science exposure (1200 s), the final flat-fielded and sky-subtracted 2-D spectrum (co-addition of 12 exposures for a total of $4 \mathrm{~h}$ ), and at the bottom the 1-D spectrum with the identification of the main absorption and emission lines (in this example an elliptical galaxy at $z=1.100$, GDS J033217.46-275234.8).

improved wavelength coverage more than compensated for the slightly comprimised flux calibration. Due to both this second order light and uncertain slit losses, we caution against using the calibrated fluxes for scientific purposes.

\section{Redshift determination}

Spectra of 399 objects have been extracted. From them we have been able to determine 234 redshifts. In the large majority of the cases the redshift has been determined through the identification of prominent features of galaxy spectra: the $4000 \AA$ break, Ca H and K, $g$-band, MgII 2798, AlII 3584 in absorption and $\mathrm{Ly}_{\alpha},[\mathrm{O} \mathrm{II}] 3727,[\mathrm{O} \mathrm{III}] 5007, \mathrm{H} \beta, \mathrm{H} \alpha$ in emission. The redshift estimation has been performed cross-correlating the observed spectrum with templates of different spectral types (S0, $\mathrm{Sa}, \mathrm{Sb}, \mathrm{Sc}$, Ell., Lyman Break, etc.), using the rusao package in the IRAF environment. The redshift identifications are summarized in Table 2.

In Table 2, the column ID contains the target identifier, that is constructed out of the target position (e.g., GDS J033206.44274728.8) where GDS stands for GOODS South. The coordinates are based on the GOODS v1.0 astrometry The quality flag, indicates the reliability of the redshift determination. Quality " $\mathrm{A}$ " indicates a solid redshift determination, "B" a likely redshift determination, "C" a tentative redshift determination and " $\mathrm{X}$ " an inconclusive spectrum or three cases in which no extraction was possible. 150 objects have been classified with quality "A", 57 with quality "B", 27 with quality "C", 69 with inconclusive redshift determination " $\mathrm{X}$ " (see Fig. 2).

The class flag groups the objects for which emission line(s) (em.), absorption-line(s) (abs.) or both (comp.) are detected in the spectrum. The classification has been guided by the observed continuum level and slope blueward and redward of the emission/absorption feature, by the broad-band colors and the morphology of the targets (see Fig. 3). 11 objects have been classified as stars.

In $38 \%$ of the cases the redshift is based only on one emission line, usually identified with [O II] 3727 or $\mathrm{Ly}_{\alpha}$. In these cases the continuum shape, the presence of breaks, the absence of other spectral features in the observed spectral range and the broad band photometry are particularly important in the evaluation. In general these solo-emission line redshifts are classified as "likely" (B) or "tentative" (C).

The comments column contains additional information relevant to the particular observation. The most common ones summarize the identification of the principal lines, the inclination of an emission line due to internal kinematics, the weakness of the signal ("faint"), the low S/N of the extracted spectrum ("noisy"), the $20 \%$ light radius ("Flux-radius" in drizzled 


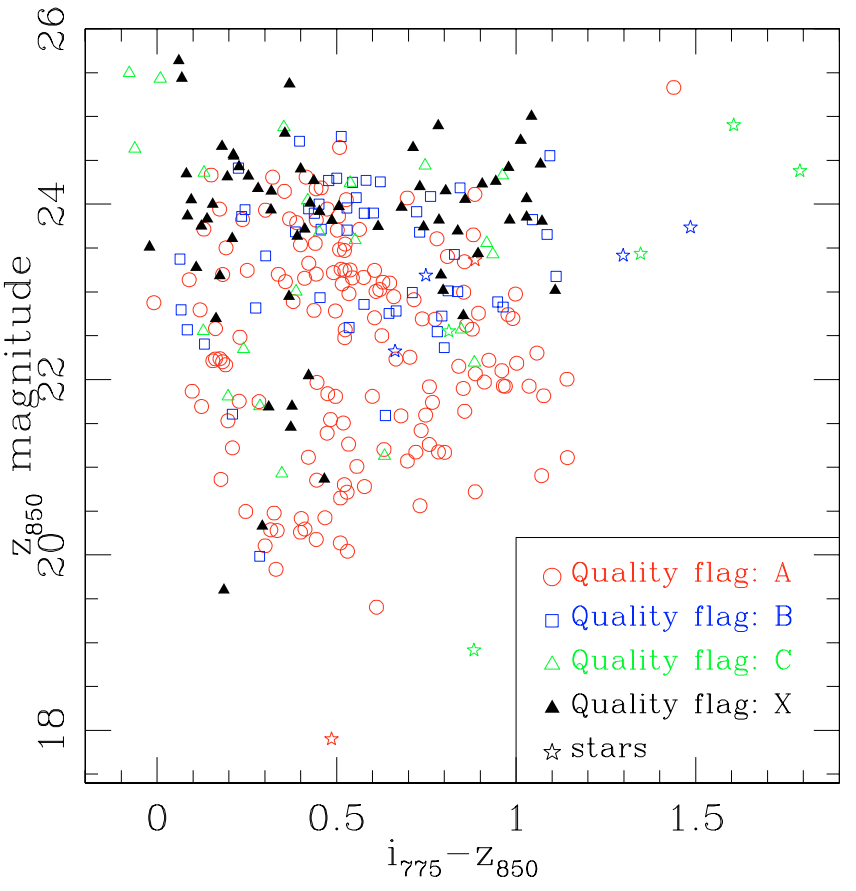

Fig. 2. Color-magnitude diagram for the spectroscopic sample as a function of the quality flag. The uncertainties in the redshift determination increase with increasing $z_{850}$ magnitude. Few bright sources (often serendipitous; $z_{850}<22$ ) have inconclusive redshift determinations due to the dithering procedure, which has positioned these sources off the slitlets for many of the exposures.

ACS pixels, 0.03 arcsecond/pixel) for objects classified as stars, etc.

Finally the column category indicates in which category described in Sect. 2 each target belongs (1 - primary catalog; 2 secondary catalog; 3 - photometric redshift sample; $4-i_{775^{-}}$ dropout and $V_{606}$-dropout; 5 - miscellaneous objects, and -1 the serendipitously identified sources).

There are two objects that are not present in the v1.0 cata$\log$, with $z=0.957$ and $z=4.882$ (marked with a cross in the Table 2). These two objects were not successfully deblended in the detection process from the brighter nearby galaxies.

The internal redshift accuracy can be estimated from a sample of 42 galaxies which have been observed twice (or more) in independent FORS2 mask sets. The distribution of measured redshift differences is presented in Fig. 4. The mean of the distribution is close to zero $\left(10^{-6}\right)$ and the redshift dispersion $\sigma_{z}=0.00078$ and mean absolute deviation $\langle|\Delta z|\rangle=0.00055$, fairly constant with redshift. These values can be considered as a lower limit to the redshift uncertainty.

\section{Discussion}

\subsection{Reliability of the redshift - comparison with VVDS}

A practical way to assess the reliability of the redshifts reported in Table 2 is to compare the present results with independent measurements of other surveys. From this point of view the recent release of the data of the VIMOS-VLT Deep Survey (VVDS, Le Fevre et al. 2004) is particularly important. There are 39 VVDS objects in common with the first release of the FORS2 GOODS survey and Fig. 5 shows the comparison of the redshift determinations. The reliability level of the redshift measurements in the VVDS is indicated by a quality flag. Flags 2-4 are the most secure with a confidence of $75 \%$, $95 \%$ and $100 \%$ respectively. Flag 1 is an indicative measurement, flag 9 indicates that there is only one secure emission line, and flag 0 indicates a measurement failure with no features identified.

For 29 cases out of $39(74 \%)$ the agreement is very good, with a mean difference $<z_{\text {FORS2 }}-z_{\text {VVDS }}>=0.0016 \pm 0.0021$.

Assuming equipartition of the redshift uncertainties between FORS2 and VVDS, we can estimate a $\sigma_{z}(\mathrm{FORS} 2) \simeq$ 0.0015 , in reasonable agreement with the estimate of Sect. 4. In the following we will assume a typical uncertainty of the redshift determinations of the present survey to be $\sigma_{z} \simeq 0.001$ (excluding "catastrophic" discrepancies).

Ten cases show "catastrophic" discrepancies, i.e. $<z_{\mathrm{FORS} 2}-$ $z_{\mathrm{VVDS}}>$ greater than 0.015 and are reported in Table 3 .

In the following we discuss case by case the origin of the discrepancy:

\section{GDS J033214.05-275124.5:}

- FORS2: the emission line [O II]3727 and the absorption lines $\mathrm{Ca} \mathrm{H}$ and $\mathrm{K}$ are detected in the FORS2 spectrum at $z=1.220$. The absorption line MgII 2798 is also present at $6210 \AA$. The $4000 \AA$ Balmer Break is also evident, quality flag "A".

- VVDS: the main emission feature in the VIMOS spectrum is identified with [O II]3727 at $z=1.325$, quality flag 3. We note an absorption feature in the VIMOS spectrum (without identification) at $\sim 6200 \AA$, consistent with the one measured in the FORS2 spectrum.

2. GDS J033219.79-274839.3:

- FORS2: flat continuum with an evident emission line at $\sim 8784 \AA$. We interpret it as [O II] 3727. No spectroscopic feature is observed at $\sim 6500 \AA$. Quality flag A.

- VVDS: the main feature in the VIMOS spectrum is identified with [O III]5007 at $z=0.568$ (emission line at $7851 \AA$ ), quality flag 2 .

3. GDS J033221.67-274056.0:

- FORS2: the emission line [O II]3727 and the absorption lines $\mathrm{Ca} \mathrm{H}$ and $\mathrm{K}$ are detected in the FORS2 spectrum at $z=1.045$, quality flag " $\mathrm{B}$ ". The [O II] 3727 line is attenuated by the sky absorption band at $\sim 7600 \AA$.

- VVDS: Ca H and K are identified in the VIMOS spectrum at $z=0.977$, quality flag 1 .

4. GDS J033226.03-275147.7:

- FORS2: the emission line [O II] 3727 (at $8356 \AA$ ) and the absorption lines $\mathrm{Ca} \mathrm{H}$ and $\mathrm{K}, \mathrm{MgI}$ and $\mathrm{B} 2630$ are detected in the FORS2 spectrum at $z=1.242$, quality flag "A".

- VVDS: the main feature is identified with $\mathrm{H} \alpha$ at $z=0.264$ (at $8296 \AA$ ), quality flag 9. No emission lines are present in the FORS2 spectrum at this wavelength.

5. GDS J033231.65-274504.8:

- FORS2: the emission line [O II]3727 and the absorption lines $\mathrm{Ca} \mathrm{H}$ and $\mathrm{K}, \mathrm{MgI}, \mathrm{H} \delta$ and are detected in the 


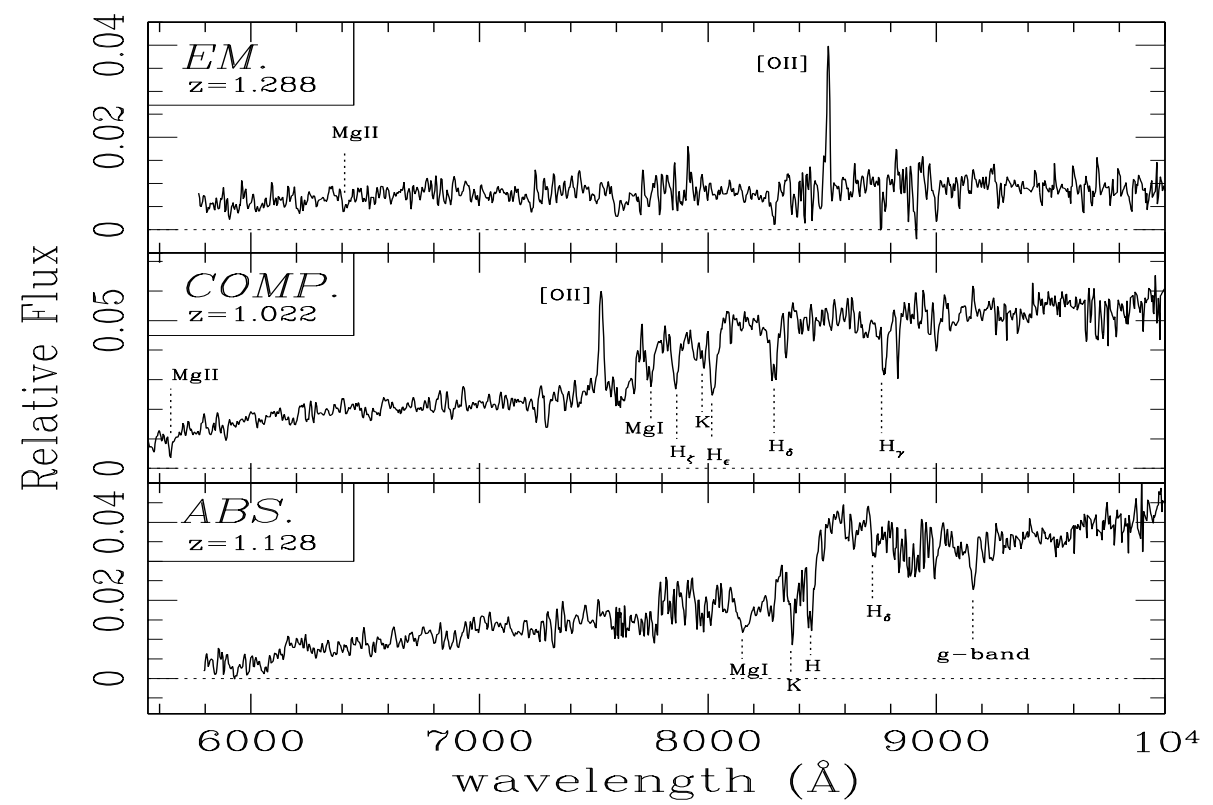

Fig. 3. Three examples of objects classified as "em." (emission-lines detected), "abs." (absorption lines) and "comp." (both emission and absorption lines detected).

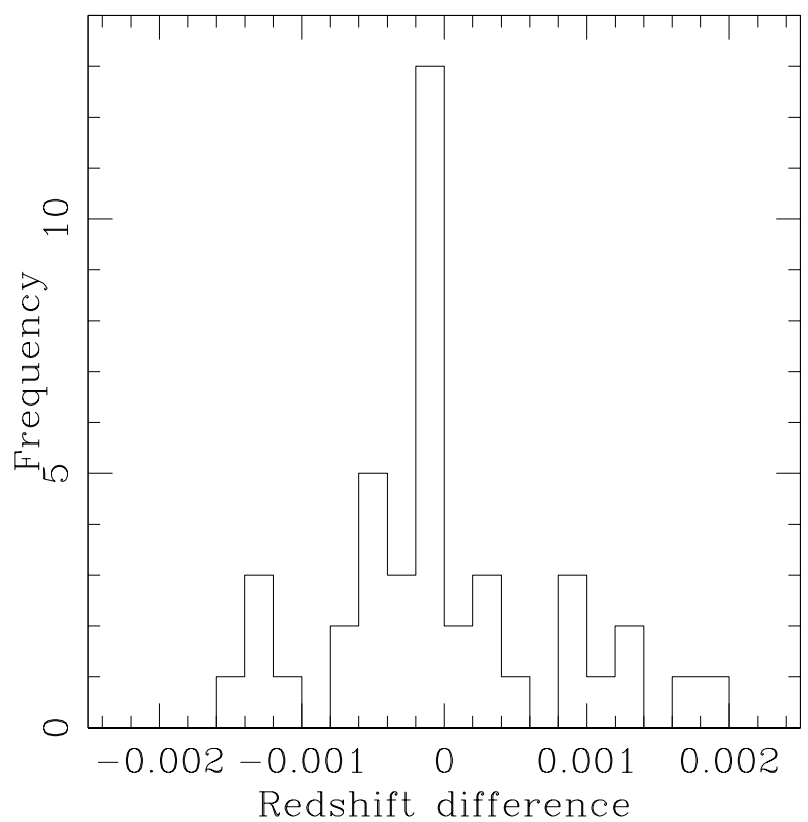

Fig. 4. Redshift differences between objects observed twice or more in independent FORS 2 observations. The distribution has a dispersion $\sigma_{z}=0.00078$.

FORS2 spectrum at $z=1.098$; the emission line $\mathrm{H} \gamma$ is also detected at $\sim 9105 \AA$, quality flag "A".

- VVDS: in the VIMOS spectrum a line is detected at $\sim 9105 \AA$, interpreted as [O II] 3727 at $z=1.443$, quality flag 1.

6. GDS J033232.32-274343.6:

- FORS2: for this object (at the border of the FORS2 field of view) the spectrum starts at $\sim 6400 \AA$. We detect a weak emission line at $\sim 7654 \AA$ (close to a sky absorption band), that we originally interpreted to be

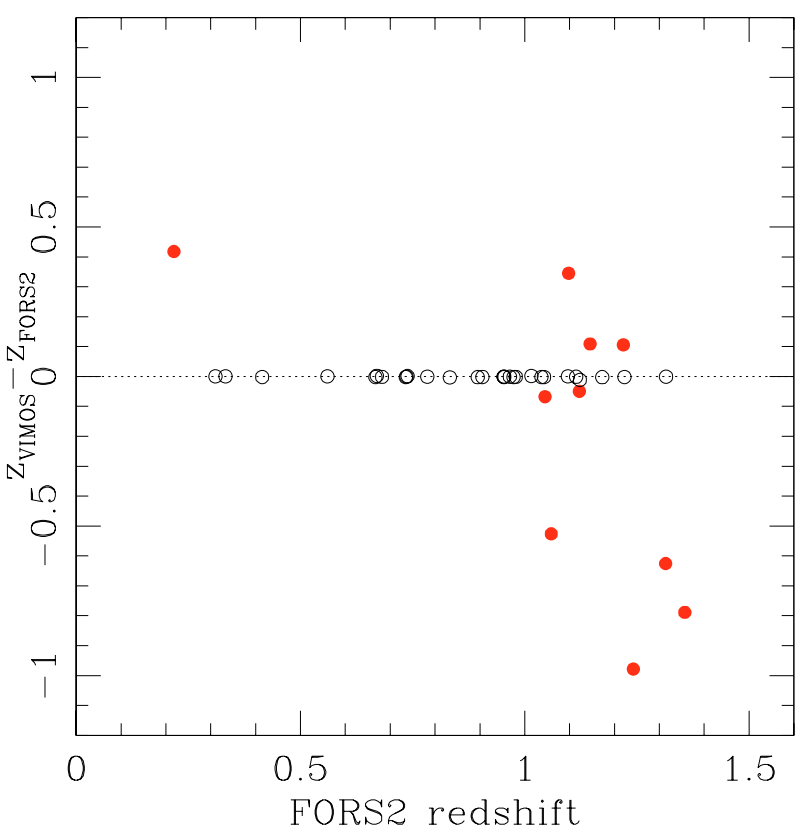

Fig. 5. VVDS spectroscopic redshift versus FORS2 spectroscopic redshift. There are 39 galaxies in common between the VVDS sample and the sample presented in this work. 10 cases show (filled symbols) discrepant redshift determination with $|\mathrm{d} z|>0.015$.

[O II]3727 at $z \sim 1.059$, assigning to the redshift a quality flag "C".

- VVDS: in the VIMOS spectrum an emission line at $\sim 5713 \AA$ is detected, interpreted as [O II]3727 at $z=0.533$ and quality flag 3 (the absorption feature $\mathrm{Ca} \mathrm{H}$ at $\sim 6085 \AA$ is also present). It is consistent with the interpretation [O II] 3727 at $z=0.533$ with the FORS2 $7654 \AA$ emission line identified as [O III]5007 
Table 3. Galaxies with discrepant redshifts between the FORS2 and VVDS surveys.

\begin{tabular}{lcccccc}
\hline \hline $\mathrm{N}$ & ID & $z$ (FORS2) & QF(FORS2) & $z$ (VVDS) & QF(VVDS) & $z$ (FORS2) $-z($ VVDS) \\
\hline 1 & GDS J033214.05-275124.5 & 1.220 & A (em.) & 1.325 & 3 & -0.105 \\
2 & GDS J033219.79-274839.3 & 1.357 & A (em.) & 0.568 & 2 & 0.789 \\
3 & GDS J033221.67-274056.0 & 1.045 & B (em.) & 0.978 & 1 & 0.067 \\
4 & GDS J033226.03-275147.7 & 1.242 & A (em.) & 0.264 & 9 & 0.978 \\
5 & GDS J033231.65-274504.8 & 1.098 & A (em.) & 1.443 & 1 & -0.350 \\
6 & GDS J033232.32-274343.6 & $1.059 \dagger$ & C (em.) & 0.533 & 3 & 0.526 \\
7 & GDS J033242.38-274707.6 & 1.314 & B (abs.) & 0.688 & 2 & 0.626 \\
8 & GDS J033242.56-274550.2 & 0.218 & A (em.) & 0.635 & 2 & -0.417 \\
9 & GDS J033249.04-275015.5 & 1.122 & B (em.) & 1.072 & 2 & 0.05 \\
10 & GDS J033249.85-274757.8 & 1.146 & B (em.) & 1.254 & 2 & -0.105 \\
\hline
\end{tabular}

$\dagger$ Adopted FORS2 value has been updated to VVDS value in Table 2.

at $z=0.533$. We have therefore updated the entry in Table 2 to a redshift $z=0.533$.

7. GDS J033242.38-274707.6:

- FORS2: this is a red object $\left(i_{775}-z_{850}=1.11\right)$, we detect two clear absorption features in the $\sim 9100 \AA$ sky free region interpreted as $\mathrm{Ca} \mathrm{H}$ and $\mathrm{K}$, faint [O II $] 3727$ seems to be present, quality flag " $\mathrm{B}$ ".

- VVDS: red spectrum, $\mathrm{Ca} \mathrm{H}$ and $\mathrm{K}$ are identified in the VIMOS spectrum at $z=0.688$, quality flag 2 .

8. GDS J033242.56-274550.2:

- FORS2: the emission lines [O III]5007 (at $6098 \AA$ ), H $\beta$ (at $5921 \AA$ ) and $\mathrm{H} \alpha$ (at $7994 \AA$ ) are detected in the FORS2 spectrum, $z=0.218$, quality flag "A".

- VVDS: the main emission feature (at $6094 \AA$ ) is identified with [O II] 3727 at $z=0.635$, quality flag 2 .

9. GDS J033249.04-275015.5:

- FORS2: the spectrum starts at $\sim 6400 \AA$. It shows continuum with a evident emission line at $\sim 7909 \AA$ interpreted as [O II] $3727(z=1.122)$, a discontinuity consistent with the $4000 \AA$ Balmer Break is present, quality flag "B".

- VVDS: the main feature in the VIMOS spectrum is an emission line at $7723 \AA$ identified with [O II]3727 at $z=1.072$, quality flag 2 .

10. GDS J033249.85-274757.8:

- FORS2: object red with bright continuum, the emission line [O II] 3727 and the absorption lines MgII 2798 and $H \zeta$ are detected in the FORS2 spectrum, the $4000 \AA$ Balmer Break is also evident, quality flag " $\mathrm{B}$ ".

- VVDS: flat continuum, the $\mathrm{NeV}$ absorption line is identified at $z=1.254$, quality flag 2 .

In summary, out of ten highly discrepant cases we have found only one that can be ascribed to an evident error in the identification of the features in the FORS2 spectrum (and the original quality flag for this object was "C"). We conclude that the probable fraction of "catastrophic" misidentifications in Table 2 is at most a few percent.

The majority of the discrepant cases appear beyond redshift 1 , for which the main spectroscopic features ([O II $] 3727$ and $\mathrm{Ca} \mathrm{H}$ and $\mathrm{K}$ ) enter the red wavelength domain, where the lower quantum efficiency and the presence of a strong fringing

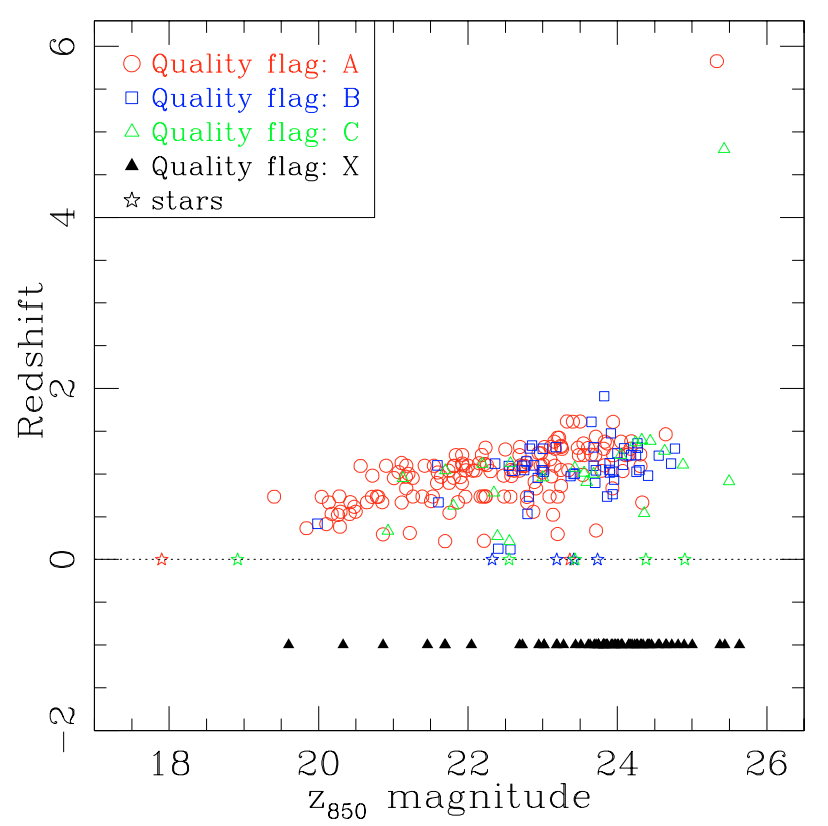

Fig. 6. Spectroscopic redshift versus magnitude for the entire FORS2 sample (quality flag "A", "B", "C" and "X"). Stars are denoted by star-like symbols at zero redshift. Inconclusive spectra are placed at $z=-1$.

pattern in the VIMOS CCD significantly affect the signal to noise ratio, and make more difficult the redshift determination.

\subsection{Reliability of the redshifts - diagnostic diagrams}

As mentioned above, the photometric information and its relation with the redshift provides useful indications about possible errors in the redshift measurement and/or magnitude estimation. The Figs. 6-8 show the redshift-magnitude, the color-redshift and the color-magnitude distributions for the spectroscopic sample (the quality flag "A" and "B" have been selected in the Figs. 7 and 8, while all sources have been plotted in the Fig. 6). In Fig. 7 the two populations of "emissionline" and "absorption-line" (typically elliptical) galaxies are clearly separated. The mean color of the "absorption-line" objects increases from $i_{775}-z_{850}=0.46 \pm 0.079$ at $\langle z\rangle=$ 0.6 to $i_{775}-z_{850}=0.86 \pm 0.18$ at $\langle z\rangle=1.0$, consistent but increasingly bluer than the colors of a non-evolving $L^{\star}$ 


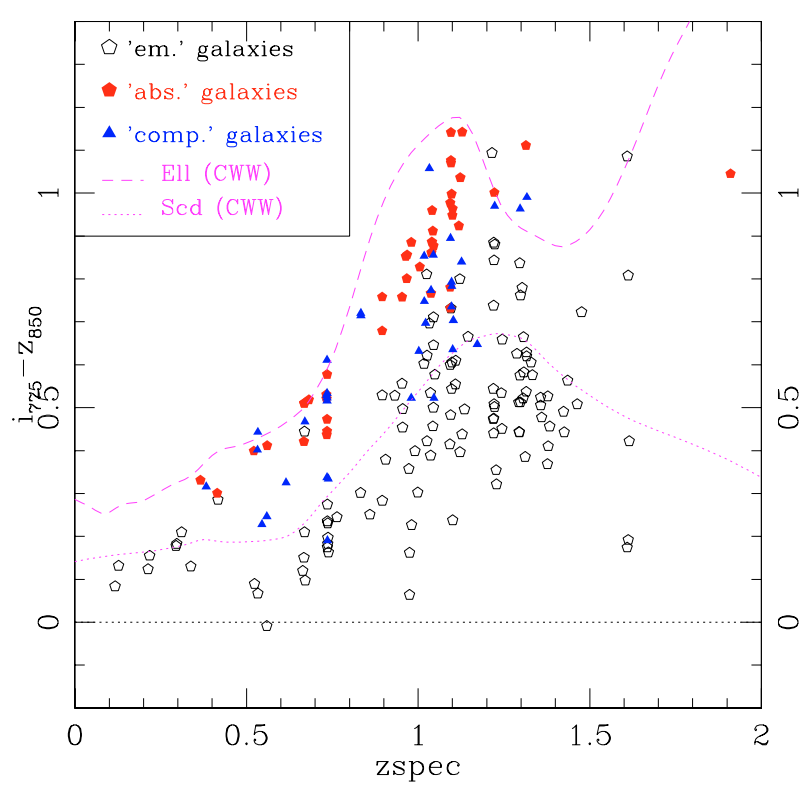

Fig. 7. Color-redshift diagram of the spectroscopic sample. Only redshifts with quality flag "A" and "B" have been selected. Filled pentagons symbols are objects identified with absorption features only ("abs." sources), while open pentagons are objects showing only emission lines ("em." sources). The intermediate cases are shown by filled triangles ("comp." sources). The long-dashed line and the short dashed line show the colors of a non-evolving $L^{\star}$ elliptical galaxy and an Scd galaxy, respectively, estimated integrating the spectral templates of Coleman et al. (1980) through the ACS bandpasses.

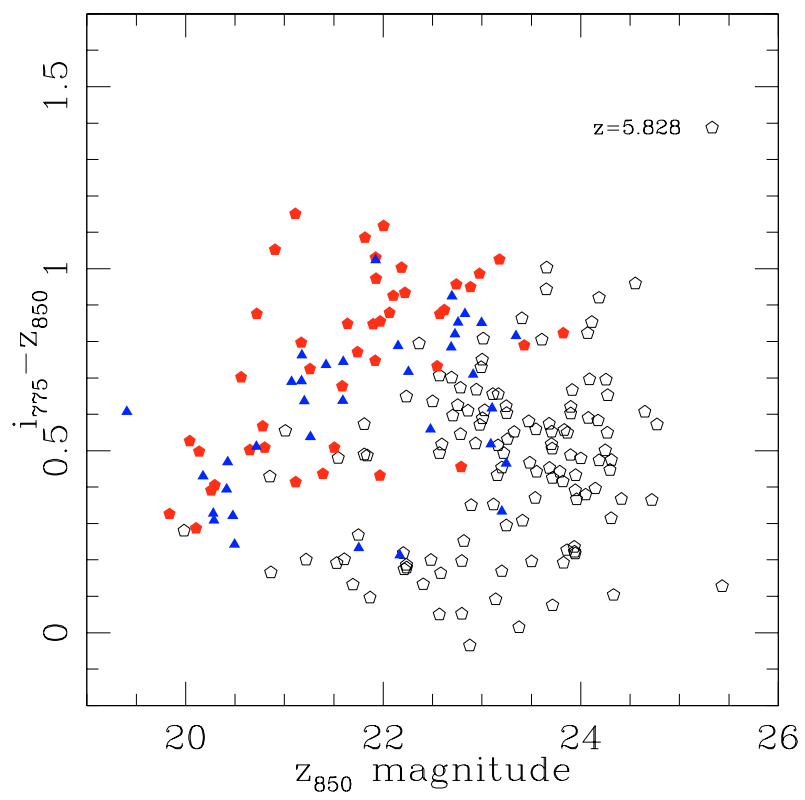

Fig. 8. Color-magnitude diagram for the spectroscopic sample. Only redshifts with quality flag "A" and "B" have been selected. The symbols are the same as in Fig. 7.

elliptical galaxy (estimated integrating the spectral templates of Coleman et al. (1980) through the ACS bandpasses).

The "emission-line" objects show in general a bluer $i_{775}-$ $z_{850}$ color and a broader distribution than the "absorption-line" sources: $i_{775}-z_{850}=0.16 \pm 0.13$ at $\langle z\rangle=0.6$ and $i_{775}-z_{850}=$ $0.52 \pm 0.21$ at $\langle z\rangle=1.1$. The broader distribution, with some

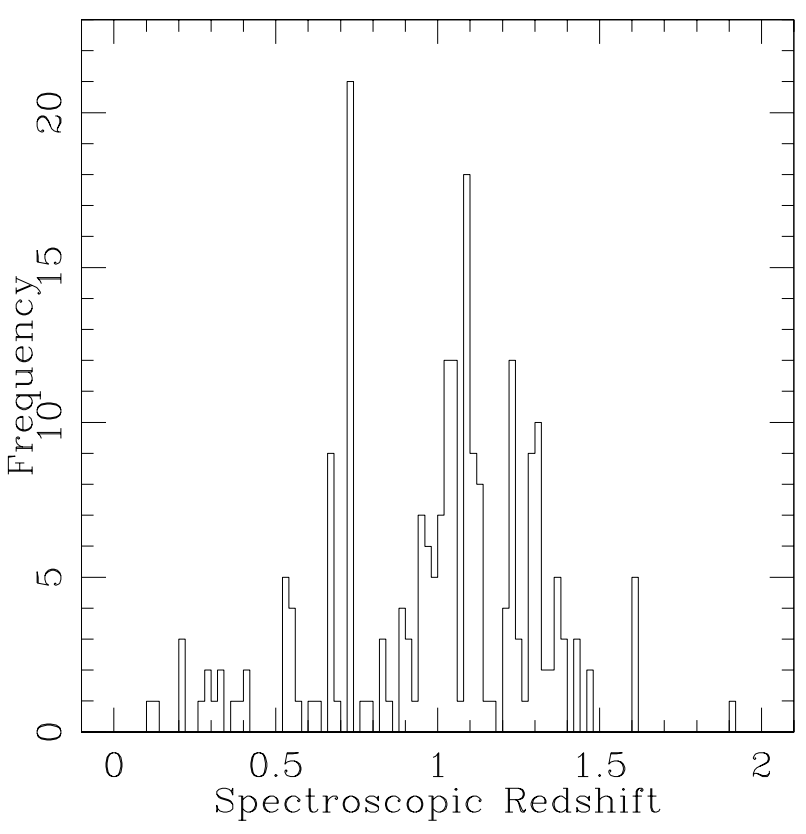

Fig. 9. Redshift distribution for the spectroscopic sample with quality A, B and C (23 redshift determinations out of 224 have quality C). Three objects at $z>4$ are not shown in the histogram.

of the "emission-line" objects entering the color regime of the ellipticals, is possibly explained by dust obscuration, high metallicity or strong line emission in the $z_{850}$ band.

\subsection{Redshift distribution and large scale structure}

Figure 9 shows the redshift distribution of the objects observed in the present survey. The majority of the sources are at redshift around $\sim 1$ (the median of the redshift distribution is at 1.04), in agreement with the main criterion for the target selection (see Sect. 2). Table 4 shows the fraction of determined redshifts as a function of the spectral features identified, i.e. emission lines, absorption lines, emission and absorption lines, and no reliable spectral features (unclassified). There are 49 galaxies identified with absorption lines only (mainly $\mathrm{Ca} \mathrm{H}$ and $\mathrm{K}$ ) in the range of redshift between 0.4-1.3; an example is shown in Fig. 1. In $46 \%$ of the total sample we have measured emission lines (mainly [O II] 3727), many of them entering the so-called "spectroscopic desert" up to $z=1.61$.

The main peaks in the redshift distribution are at $z \sim 0.73$ (21 galaxies) and 1.1 (25 galaxies). Two concentrations at $z \sim$ 1.6 (with 5 galaxies at the mean redshift $\langle z\rangle=1.612 \pm 0.003$, see the two dimensional spectra in Fig. 11) and $z \sim 0.67$ (9 galaxies) are also apparent. The presence in the CDF-S of large scale structure, (LSS) at $z \sim 0.73$ and $z \sim 0.67$ is already known (Cimatti et al. 2002; Gilli et al. 2003; Le Fevre et al. 2004). The peak at $z \sim 1.1$ seems to be a new indication of large scale structure, of the 25 galaxies in the range $1.09<z<1.11,10$ show emission lines, 9 are ellipticals and 6 are intermediatetype galaxies.

The significance of the LSS at $z=1.61$ is confirmed by:

1. the observations of Gilli et al. (2003) who found a peak in the redshift distribution of X-ray sources at $z=1.618$ 
Table 4. Fractions of sources with different spectral features. The fractions of the different categories observed in Sect. 2 are also shown.

\begin{tabular}{lcccccccccc}
\hline \hline Spectral class & $z_{\operatorname{mean}}$ & $z_{\min }$ & $z_{\max }$ & Total fraction & Cat.1) & Cat.2) & Cat.3) & Cat.4) & Cat.5) & Cat.-1) (seren.) \\
\hline Emission & 1.131 & 0.117 & 5.828 & $45.6 \%$ & $11.9 \%$ & $6.7 \%$ & $9.2 \%$ & $1.7 \%$ & $0.3 \%$ & $15.8 \%$ \\
Absorption & 0.950 & 0.366 & 1.910 & $16.2 \%$ & $9.6 \%$ & $1.0 \%$ & $0.0 \%$ & $0.3 \%$ & $0.0 \%$ & $5.3 \%$ \\
Em. \& abs. & 0.897 & 0.382 & 1.317 & $12.3 \%$ & $4.6 \%$ & $1.7 \%$ & $1.3 \%$ & $0.3 \%$ & $0.0 \%$ & $4.4 \%$ \\
Stars & 0.000 & 0.000 & 0.000 & $3.7 \%$ & $1.0 \%$ & $0.0 \%$ & $0.0 \%$ & $2.0 \%$ & $0.0 \%$ & $0.7 \%$ \\
Unclassified & - & - & - & $22.2 \%$ & $4.6 \%$ & $1.7 \%$ & $3.6 \%$ & $4.6 \%$ & $1.0 \%$ & $6.7 \%$ \\
\hline
\end{tabular}

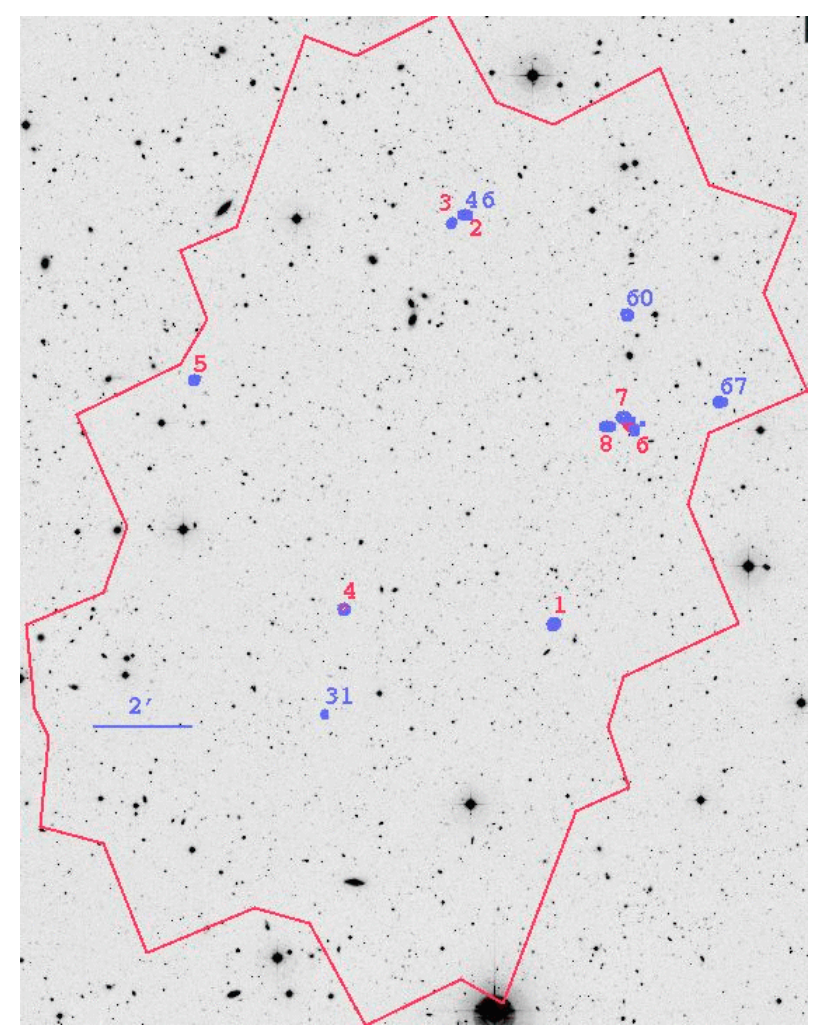

Fig. 10. The spatial distribution of the galaxies at $z \sim 1.61$ in the CDF-S. The background image is an exposure in the $R$ band obtained with the ESO-WFI instrument, north is up and east on the left. The red contour outline the ACS GOODS-S field and the horizontal bar indicates a 2 arcmin size (corresponding to $1020 \mathrm{kpc}$ at $z=1.61$ ). The five $z=1.61$ galaxies discovered in the present work are marked as 1, 2, 3, 4 and 5, corresponding to J GDS033217.31275025.0, J GDS033225.10-274219.5, J GDS033226.40-274228.2, J GDS033236.04-275004.3, and J GDS033249.49-274534.2, respectively. The three K20 sources are shown with the numbers 6,7 and 8 . The numbers $31,46,60,67$, show the positions of the $z \sim 1.61 \mathrm{X}$-ray sources (see text).

(5 galaxies) and measured a Poissonian probability of $3.8 \times$ $10^{-3}$ for a chance distribution;

2. three more galaxies at $z=1.605,1.610,1.615$ in the K20 survey Cimatti et al. (2002).

The structure at $z \approx 1.61$ is extending across a transverse size of $\sim 5 \mathrm{Mpc}$ in a wall-like pattern rather than a group structure (see Fig. 10).

\subsection{High redshift galaxies}

As discussed in Sect. 2, the target selection includes mainly low redshift objects $(z<2)$. For three galaxies, however, a redshift larger than four was measured: the galaxy GDS J033240.01-274815.0 at $z=5.828$ the only $i_{775^{-}}$ dropout (see Sect. 2) actually targeted in the present observations and two serendipitously-observed high redshift sources, GDS J033228.84 - 274132.7 and one object at $\alpha=$ $3^{\mathrm{h}} 32^{\mathrm{m}} 28.94^{\mathrm{s}}, \delta=-27^{\circ} 41^{\prime} 28.19^{\prime \prime}$ not present in the cata$\log \mathrm{v} 1.0$, measured at $z=4.800$ and $z=4.882$, respectively.

The $i$-dropout candidate has been also selected by Stanway et al. (2003) (identified as SBM03\#1) and confirmed as a $z=5.83 \mathrm{Ly}_{\alpha}$ emitter by Stanway et al. (2004) (as SBM03\#1 and GLARE\#1042), Dickinson et al. (2004a) (as SiD002) and the present work (as GDS J033240.01-274815.0). In Fig. 12 the FORS 2 spectrum of the $i_{775}$-dropout source is shown. The $\mathrm{Ly}_{\alpha}$ line is clearly detected at $z=5.828$ and shows the blue cut-off characteristic of high-redshift $\mathrm{Ly}_{\alpha}$ emitters and the $\mathrm{Ly}_{\alpha}$ forest continuum break. It is interesting to note (Fig. 2) that out of the six reddest $i_{775}-z_{850}$ objects targeted, five turned out to be stars and only one a $z=5.83$ galaxy. This indicates that for the bright end of the $i_{775}$-dropouts the contaminant fraction of low-mass stars is large. However, the ACS imaging provides a robust measure of stellarity, at least down to $z_{850} \simeq 26$. At fainter magnitudes (as shown in Bunker et al. 2004 in the Hubble Ultra Deep Field) the stellar contamination in the faint end of the $i_{775}$-dropouts distribution (down to $z_{850} \simeq 28.5$ ) is only $2 \%$ and the majority of the candidates are spatially resolved.

Figure 13 shows a peculiar system of three sources: two emission-line sources above ( $\sim 1.5$ arcsecond) and below ( $\sim 3$ arcsecond) the main galaxy GDS J033228.88-274129.3, clearly visible in the ACS color image and in the two dimensional spectrum. The same target has been observed in two different masks adopting the same orientation of the slits. The total exposure time is $\simeq 43 \mathrm{ks}$. The extracted one dimensional spectra are shown in the right side of the Fig. 13.

The main galaxy GDS J033228.88-274129.3 has a redshift $z=0.733$ with both emission and absorption lines measured (quality flag "A"): [O II]3727, MgI, Ca H and K, g-band, etc. The bottom object (GDS J033228.84-274132.7) shows a soloemission line at $7052 \AA$ (see the 1-D spectrum), and is not detected in the ACS B band, we interpret this line as $\mathrm{Ly}_{\alpha}$ at $z=4.800$ with quality " $C$ ".

The source above GDS J033228.88-274129.3 is most probably a $\mathrm{Ly}_{\alpha}$ emitter at redshift $z=4.882$ (quality "B"). The spectrum has been extracted subtracting the contamination of the tail of the main galaxy. After the subtraction the shape of 


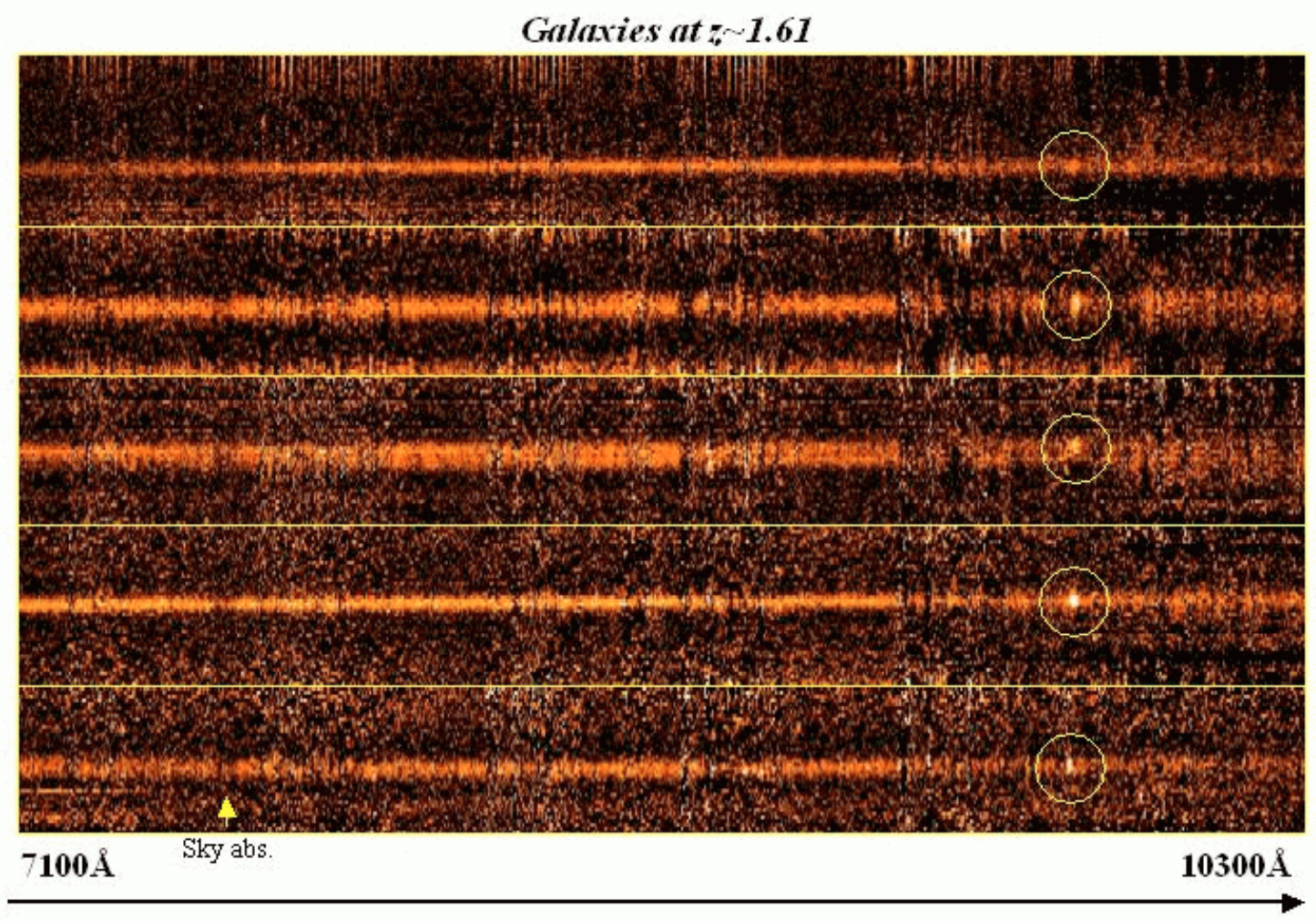

Fig. 11. Two dimensional spectra of 5 galaxies at $z=1.61$. The [O II $] 3727$ emission line is marked with a circle at $9727.5 \AA$. The absorption sky feature ( $7600 \AA, A$ band) is indicated with an arrow. It is worth to note the optimal red sensitivity of FORS2.

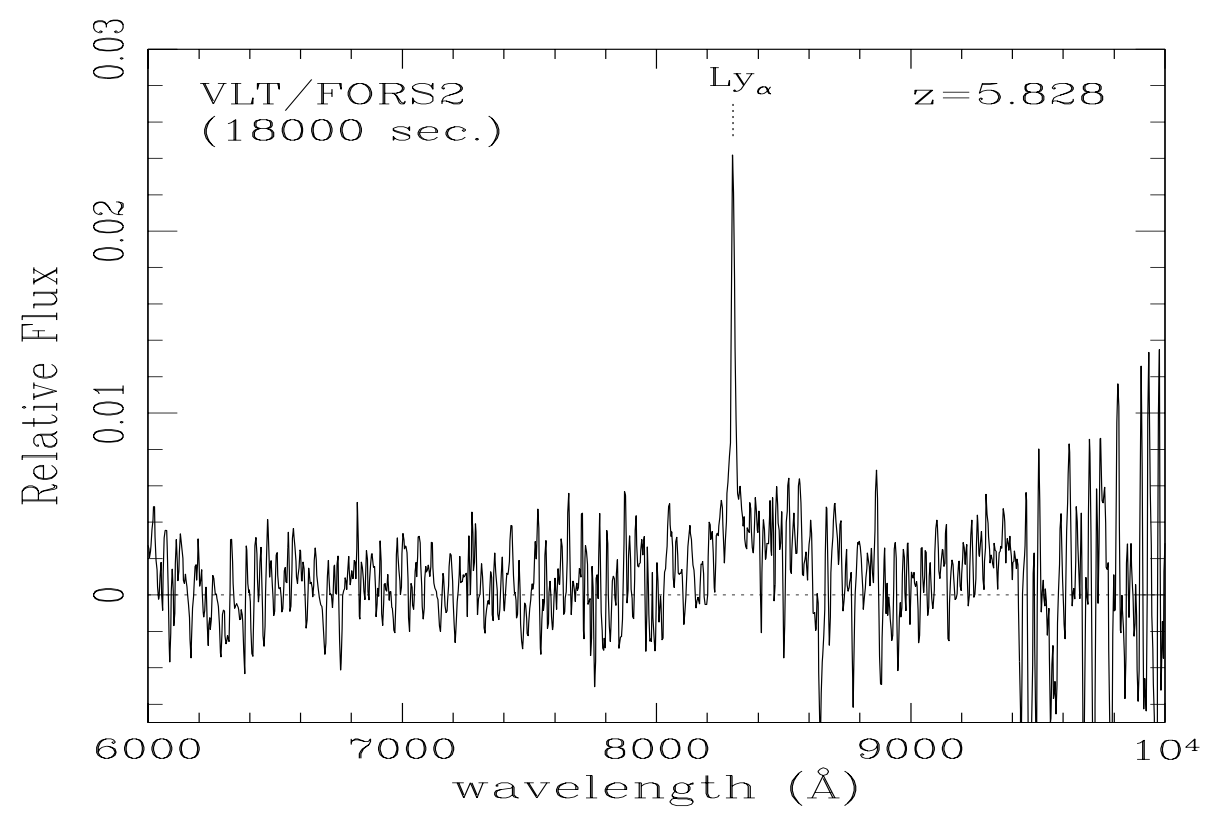

Fig. 12. VLT spectrum of the $i_{775}$-dropout galaxy GDS J033240.01-274815.0.

the spectrum shows the blue cut-off and the $\mathrm{Ly}_{\alpha}$ forest continuum break, typical of the LBGs.

\subsection{Dynamical masses of galaxies at $z \sim 1$}

Three galaxies, GDS J033215.88-274723.1, GDS J033225.86275019.7 and GDS J033230.71-274617.2, at redshift $z=0.896,1.095$ and $z=1.307$ respectively show a spatially resolved [O II]3727 line with a characteristic "tilt" indicative of a high rotation velocity (see Fig. 14).

Various studies have been carried out on the internal kinematics of distant galaxies (Vogt at al. 1996, 1997; Moorwood et al. 2001; Pettini et al. 2001; van Dokkum \& Stanford 2001). Rigopoulou et al. 2002 have determined velocity profiles with a medium resolution grating $R \sim 5000$ of three galaxies at $z \sim 0.6$ and one at $z \sim 0.8$, detected by ISOCAM in the 


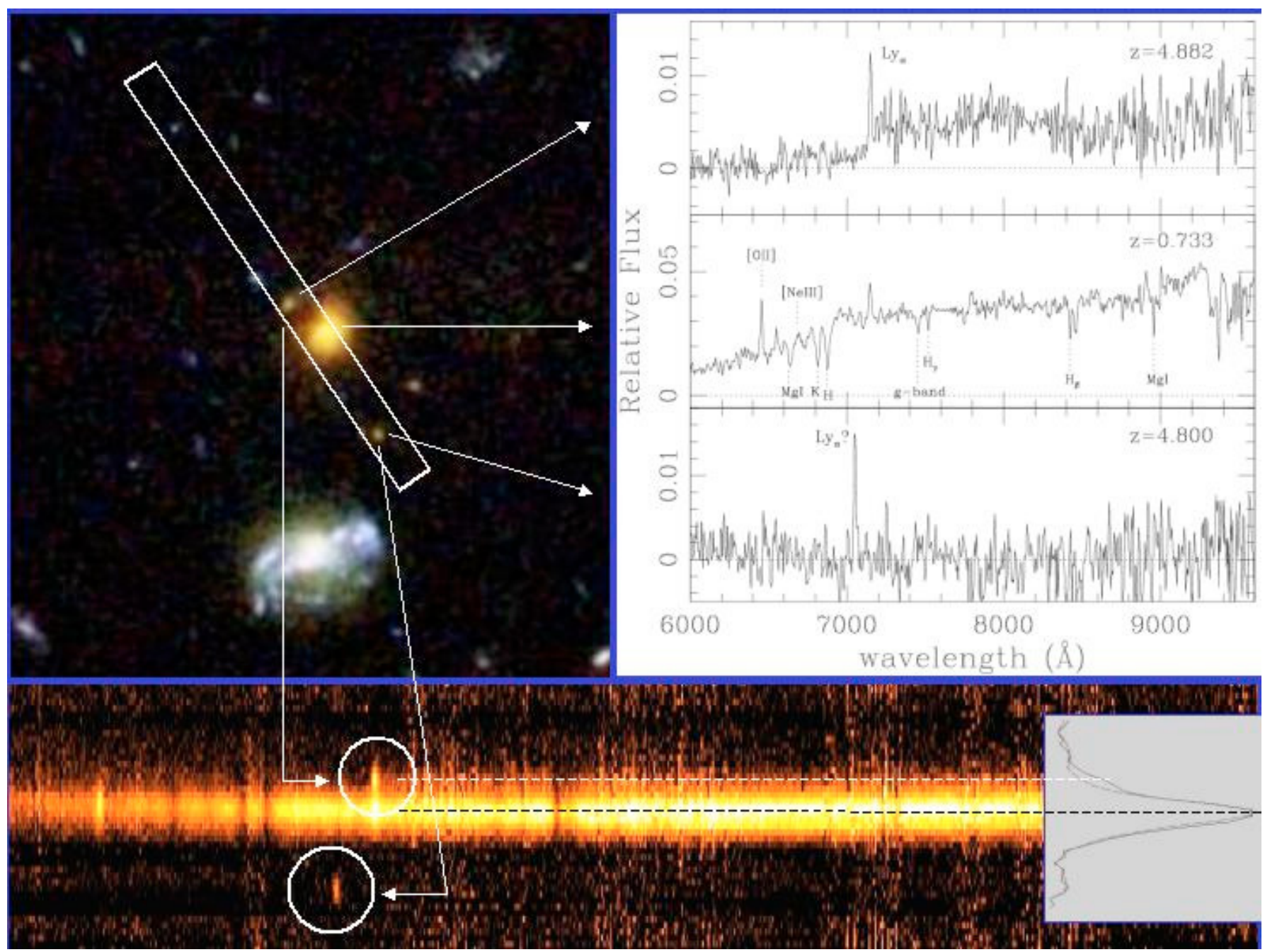

Fig. 13. Simultaneous spectrum of three sources in the slit. On the right of the figure, the $1 \mathrm{D}$ spectra of the $z=0.733$ main galaxy GDS J033228.88-274129.3, the single emission line $\sim 3$ arcsec below (GDS J033228.84-274132.7) and the object $\sim 1.5$ arcsec above are shown. The left-hand panel shows the ACS color image, 5 arcsec on a side. North is up, east is to the left. The bottom panel shows the 2D spectrum, with the spatial profile obtained by collapsing 80 columns ( $256 \AA$ ), centered at $7150 \AA$, shown to the right. Candidate serendipitous $\mathrm{Ly}_{\alpha}$ emission lines are clearly marked. The object above the target source shows faint continuum reward of the emission line.

HDF-S. For one object they have derived a rotational velocity of $460 \mathrm{~km} \mathrm{~s}^{-1}$ containing a mass of $10^{12} M_{\odot}$ (within a radius of $20 \mathrm{kpc}$ ) significantly higher than the dynamical masses measured in most other local and high redshift spirals.

In the case of GDS J033215.88-274723.1, GDS J033225.86-275019.7 and GDS J033230.71-274617.2, the spectra, in spite of the relatively low resolution $\mathfrak{R} \sim 860$, clearly show a tilt of several pixels (corresponding to about $10 \AA$ ). The measured velocity increases with increasing distance from the center of the objects reaching a value of the order of and greater than $400 \mathrm{~km} \mathrm{~s}^{-1}$ at the extremes. For the object GDS J033225.86-275019.7 we have measured a displacement between the two extreme peaks of $11.5 \AA$ (top panel of the Fig. 14), while a displacement of $9.6 \AA$ has been measured in the case of GDS J033215.88-274723.1 (middle panel of the Fig. 14)

Assuming that the observed velocity structure is due to dynamically-relaxed rotation, then it is possible to estimate the dynamical mass for the three galaxies shown in Fig. 14 (e.g. Lequeux 1983): $\frac{1.6}{\sin ^{2}(i)} \times 10^{11} M_{\odot}$ for the galaxy GDS J033215.88-274723.1 (within a radius of $7.8 \mathrm{kpc}$ ) and $\frac{3.1}{\sin ^{2}(i)} \times 10^{11} M_{\odot}$ for the galaxy GDS J033225.86-275019.7 (within a radius of $9.8 \mathrm{kpc}$ ). The noisy spectrum of the galaxy GDS J033230.71-274617.2 allows us to roughly measure a dynamical mass of the order of $\frac{1.5}{\sin ^{2}(i)} \times 10^{11} M_{\odot}$ (within a radius of $7.5 \mathrm{kpc}$ ). The estimates should be considered a lower limit to the total dynamical mass because more external parts of the rotating structure might have a lower surface brightness and remain undetected.

\subsection{GDS J033210.93-274721.5: A spectrum contaminated by a nearby galaxy}

The spectrum of the galaxy GDS J033210.93-274721.5 simultaneously shows features corresponding to the redshifts $z=1.222$ and $z=0.417$ (Fig. 15). The origin of the overlap 

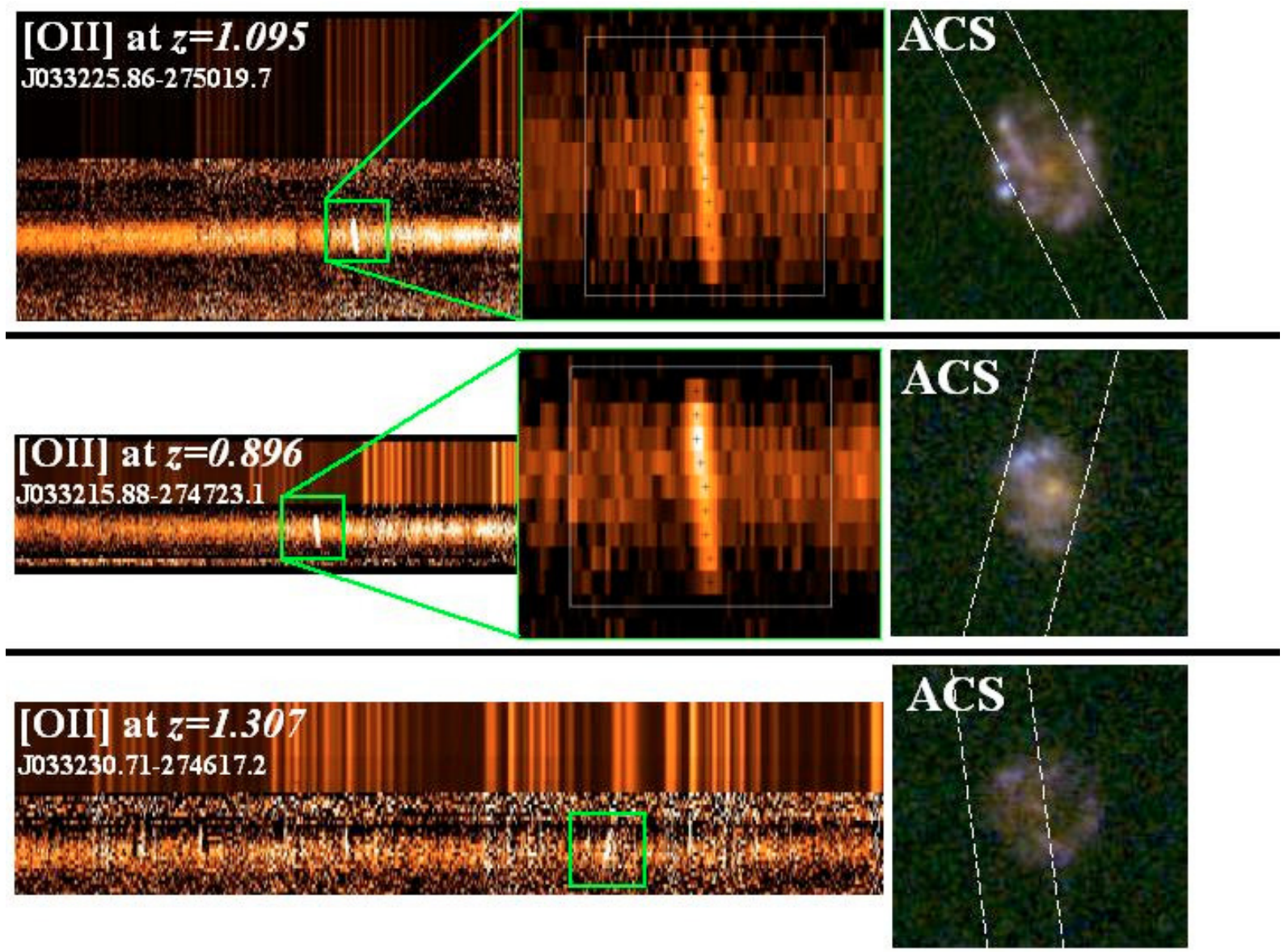

Fig. 14. Three examples of tilted [O II] 3727 emission line at redshift around 1. The two dimensional FORS2 spectra are shown (object and sky lines). In the first two spectra (top and middle) a zoom of the [O II] 3727 emission line is shown (the white rectangle underline the region where the Gaussian fit has been performed to derive the line peak, small black crosses), in the bottom spectrum the line is too faint to calculate a reliable peak (this object has been serendipitously-identified). In the right side of the spectra the ACS images of the galaxies and the slits orientation are shown.

is the presence of a nearby galaxy $\left(z_{850}=19.98\right.$, GDS J033210.92-274722.8) offset by 1.3 arcsecond with a redshift $z=0.417$. Light from the brighter $z=0.417$ galaxy contaminates the spectrum of the fainter $\left(z_{850}=22.19\right)$, higher redshift galaxy GDS J033210.93-274721.5 (see Fig. 15). Such cases may represent a problem and a source of error in large spectroscopic surveys, which require an highly automated data processing. A possible solution is to evaluate a priori on the basis of imaging what are the cases subject of light contamination requiring a "special" reduction. Alternatively, colorredshift diagrams (such as Fig. 7), a comparison of spectroscopic and photometric redshifts or similar diagnostics are required to carry out the necessary data quality control and identify possible misidentifications.

\section{Conclusions}

In the framework of the Great Observatories Origins Deep Survey a large sample of galaxies in the Chandra Deep Field South has been spectroscopically targeted. A total of $303 \mathrm{ob}-$ jects with $z_{850} \lesssim 25.5$ has been observed with the FORS2 spectrograph at the ESO VLT providing 234 redshift determinations. From a variety of diagnostics the measurement of the redshifts appears to be highly accurate (with a typical $\sigma_{z}=0.001$ ) and reliable (with an estimated rate of catastrophic misidentifications at most few percent). The reduced spectra and the derived redshifts are released to the community (http://www. eso.org/science/goods/). They constitute an essential contribution to reach the scientific goals of GOODS, providing the time coordinate needed to delineate the evolution of galaxy masses, morphologies, and star formation, calibrating the photometric redshifts that can be derived from the imaging data at $0.36-8 \mu \mathrm{m}$ and enabling detailed studies of the physical diagnostics for galaxies in the GOODS field.

Acknowledgements. We are grateful to the ESO staff in Paranal and Garching who greatly helped in the development of this programme. The work of DS was carried out at the Jet Propulsion Laboratory, California Institute of Technology, under a contract with NASA. L.A.M. acknowledges support by NASA through contract number 1224666 issued by the Jet Propulsion Laboratory, California Institute of Technology under NASA contract 1407 . We thank the ASI grant $\mathrm{I} / \mathrm{R} / 088 / 02$ (SC, MN, EV). 

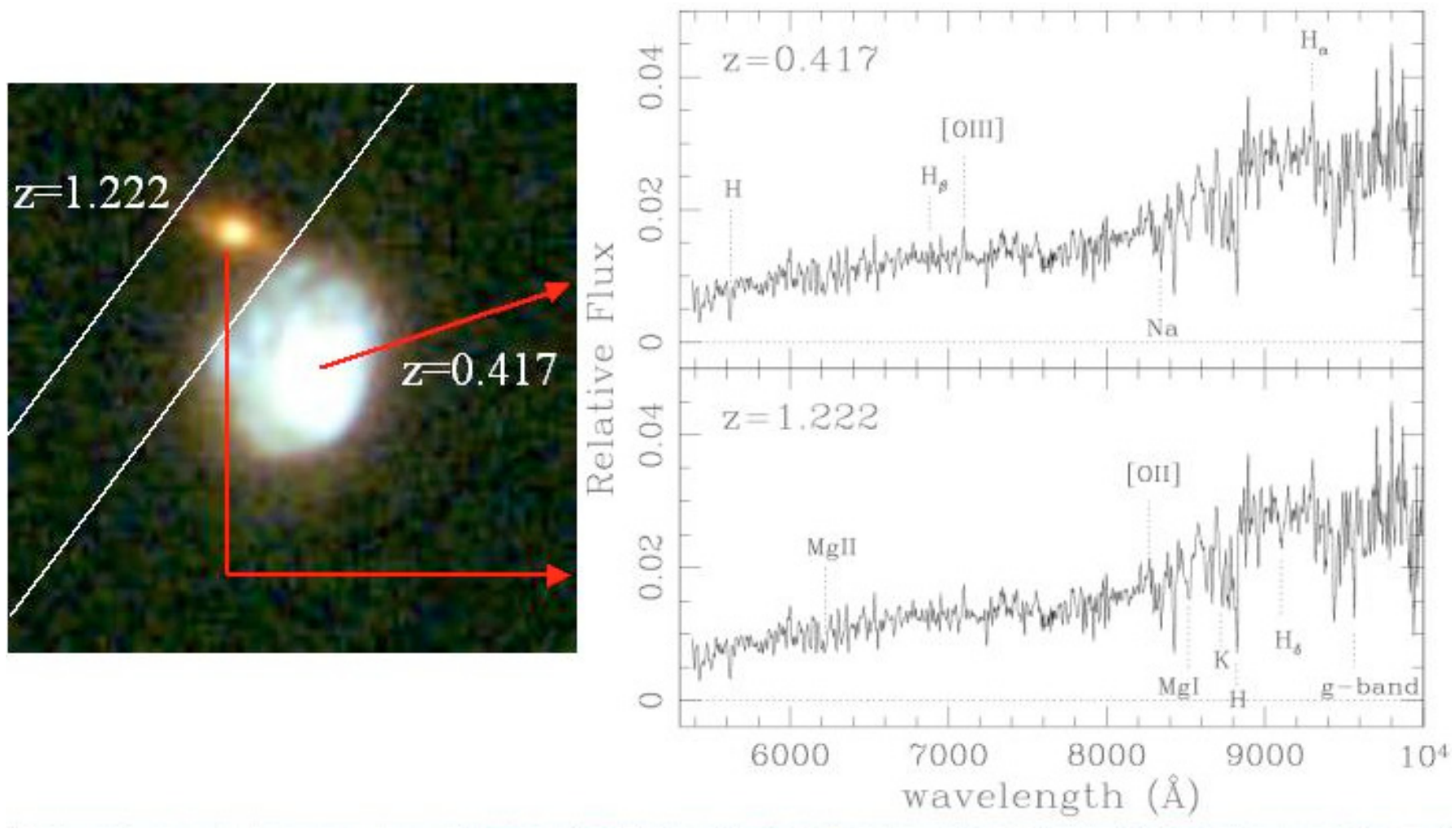

Fig. 15. The light merged case, two objects at different redshift superimposed in the slit (marked with white lines in the left panel). In the right panel the same extracted spectrum with different identifications. An elliptical galaxy (the target, GDS J033210.93-274721.5) at $z=1.222$ clearly identified with the $\mathrm{Ca} \mathrm{H}$ and $\mathrm{K}, \mathrm{H} \delta, \mathrm{MgI}$ (quality flag "A"). The bright bluer object (GDS J033210.92-274722.8) shows absorption and emission lines: $\mathrm{Ca} \mathrm{H},[\mathrm{O}$ III] $5007, \mathrm{Na}, \mathrm{H} \alpha$ at $z=0.417$ (quality flag “B”). The $\mathrm{Ca} \mathrm{K}$ is contaminated by the sky line $\sim 5577 \AA$.

\section{References}

Abraham, R. G., van den Bergh, S., Glazebrook, K., et al. 1996, ApJ, 107,1

Bahcall, J. N., Schmidt, M., \& Soneira, R. M. 1982, ApJ, 258, 17

Bohlin, R. C., Colina, L., \& Finley, D. S. 1995, AJ, 110, 1316

Bunker, A. J., Stanway, E. R., Ellis, R. S., \& McMahon, R. G. 2004, AAS, 204, 9103

Cimatti, A., Mignoli, M., Daddi, E., et al. 2002, A\&A, 392, 395

Coleman, G. D., Wu, C.-C., \& Weedman, D. W. 1980, ApJS, 43, 393

Dickinson, et al. 2003, in Proc. of the ESO/USM Workshop "The Mass of Galaxies at Low and High Redshift" (Venice, Italy, October 2001), ed. R. Bender, \& A. Renzini [arXiv: astro-ph/0204213]

Dickinson, M., Stern, D., Giavalisco, M., et al. 2004, ApJ, 600, 99

Giavalisco, M., Ferguson, H. C., Koekemoer, A. M., et al. 2004, ApJ, 600, L93

Giavalisco, M., Dickinson, M., Ferguson, H. C., et al. 2004, ApJ, 600, 103

Gilli, R., Cimatti, A., Daddi, E., et al. 2003, ApJ, 592, 721

Le Fevre, O., Vettolani, G., Paltani, S., et al. and the VIMOS VLT Deep Survey team, A\&A, 428, 1043

Lequeux, J. 1983, A\&A, 125, 394

Moorwood, A. F. M., van der Werf, P. P., Cuby, J. G., \& Oliva, E. 2000, A\&A, 362, 9
Mobasher, B., Idzi, R., Benítez, N., et al. 2004, ApJ, 600, 167

Moustakas, L. A., Casertano, S., Conselice, C. J., et al. 2004, ApJ, 600,131

Oke, J. B., Cohen, J. G., Carr, M., et al. 1995, PASP, 107, 375

Pettini, M., Shapley, A. E., Steidel, C. C., et al. 2001, ApJ, 588, 65

Renzini, et al. 2002, in Proc. of the ESO/USM Workshop "The Mass of Galaxies at Low and High Redshift" (Venice, Italy, October 2001), ed. R. Bender, \& A. Renzini

Riess, A. G., Strolger, L.-G., Tonry, J., et al. 2004, ApJ, 607, 665

Rigopoulou, D., Franceschini, A., Aussel, H., et al. 2002, ApJ, 580, 789

Stanway, E. R., Bunker, A. J., McMahon, R. G., et al. 2004, ApJ, 607, 704

Stanway, E. R., Bunker, A. J., \& McMahon, R. G. 2003, MNRAS, 342,439

Steidel, C. C., Adelberger, K. L., Giavalisco, M., Dickinson, M., \& Pettini, M. 1999, ApJ, 519, 1

Szokoly, G. P., Bergeron, J., Hasinger, G., et al. 2004 [arXiv:astro-ph/0312324]

van Dokkum, P. G., \& Stanford, S. A. 2001, ApJ, 562, 35

Vogt, P. N., Forbes, D. A., Phillips, A. C., et al. 1996, ApJ, 465, L15

Vogt, P. N., Phillips, A. C., Faber, S. M., et al. 1996, ApJ, 479, L121

Warmels, R. H. 1991, The ESO-MIDAS System, in Astronomical Data Analysis Software and Systems I , PASP Conf. Ser., 25, 115 